\title{
Malaria prevention, diagnosis and treatment strategies in Kaduna State, Nigeria
}

\author{
Obafemi J. Babalola*1, 2 \\ ${ }^{1}$ Residence Cohort VI, Nigeria Field Epidemiology and Laboratory training pro, KADUNA STATE, Nigeria; ${ }^{2}$ Federal Neuropsychiatric \\ Hospital, KADUNA STATE, Nigeria
}

\section{Objective}

We aim to assess the implementation of malaria prevention, diagnosis and treatment strategies, to assess implementation trends from 2011 to 2014 and if surveillance targets were met.

\section{Introduction}

Malaria is a preventable disease but 3.4 billion people at risk globally with 207 million cases and 627 deaths reported in 2013. Africa accounts for $80 \%$ of cases and $90 \%$ of all malaria deaths. Nigeria accounts for $25 \%$ of malaria burden in Africa. The goal of malaria control is to reduce malaria -related transmissions, cases and deaths to a level where it is no longer a public health concern

\section{Methods}

Kaduna state, north western Nigeria with estimated population of 7.3 million has 23 districts and 1252 health facilities. Of these 461 sent malaria surveillance data to National Health Management Information System monthly. Data from January 2011 to December 2014 was analysed. We evaluated variables related to malaria interventions strategies such as malaria diagnosis, malaria treatment, malaria prevention in pregnancy. Frequencies, proportions and trend analysis were done and odd ratios for associations between variables were calculated with confidence interval set at 95\%. Epi info statistical software was used for the analysis.

\section{Results}

Data completeness was $89.8 \%$. Of the $1,008,728$ people that visited health facilities, $56.6 \%$ presented with fever. Among the fever cases, $34.2 \%$ was tested with rapid diagnostic test (RDT) and $5.5 \%$ with microscopy. Artemisinin based combination therapy was given to 361,464 of which $36.4 \%$ had confirmed malaria. Those aged $<5$ years with suspected fever were 1.28 (95\% confidential interval (C.I), 1.27-1.29; $\mathrm{p}<0.01)$ ) more likely not to be tested with either RDT or microscopy and they are 2.62 (95\% C.I., $2.63-6.67$; $\mathrm{p}<0.01$ ) times more likely to have ACT for confirmed malaria. ACT prescription to presumptive malaria increases from $31.8 \%$ in 2013 to $200.2 \%$ in 2014 . There is a progressive increase of long lasting insecticidal net distribution and access to second dose of Intermittent Preventive Therapy (IPT-2) for pregnant women.

\section{Conclusions}

Generally, progress in Malaria control transition to Elimination in Kaduna State, Nigeria is favorable with malaria prevalence at $36.4 \%$. Some targets were met within the period and recommend strengthening of these malaria control strategies with focus on vulnerable groups and prevent uncontrolled ACT prescription for presumptive malaria.

\section{Keywords}

Malaria surveillance; Malaria control strategy; Malaria elimination program; Malaria in pregnancy,; Rapid diagnostic test

\section{Acknowledgments}

We acknowledge Kaduna State Ministry of Health, Kaduna Nigeria, Kaduna State Malaria Elimination Program and Nigeria Field Epidemiology and Laboratory Training Program, FCT, Abuja, Nigeria

\section{*Obafemi J. Babalola}

E-mail: drfemibabs@yahoo.com 